\title{
Phase Transformation Mechanism of Amorphous Calcium Phosphate to Hydroxyapatite Investigated by Liquid-Cell Transmission Electron Microscopy
}

Biao Jin ${ }^{\dagger,}$, Zhaoming Liu ${ }^{\dagger}$, Changyu Shao ${ }^{\dagger}$, Jiajun Chen ${ }^{\dagger}$, Lili Liü, Ruikang Tang ${ }^{*}$, , and James J De

$$
\text { Yoreo }{ }^{*} \text { t, } \$
$$

'Department of Chemistry, Zhejiang University, Hangzhou, Zhejiang 310027, China

† Physical Sciences Division, Pacific Northwest National Laboratory, Richland, Washington 99352, USA.

${ }^{\S}$ Department of Materials Science and Engineering, University of Washington, Seattle, Washington 98195, USA

\section{Corresponding Authors}

*E-mail: James.DeYoreo@pnnl.gov

*E-mail: rtang@zju.edu.cn 


\section{Supporting Figures}


Figure S1. (a) Cryo-TEM images of ACP at the early stage of formation and transformation. (b) The FTIR spectrum of ACP nanoparticles in the presence of $0.3 \mathrm{mM} \mathrm{Mg}^{2+}$ ions.
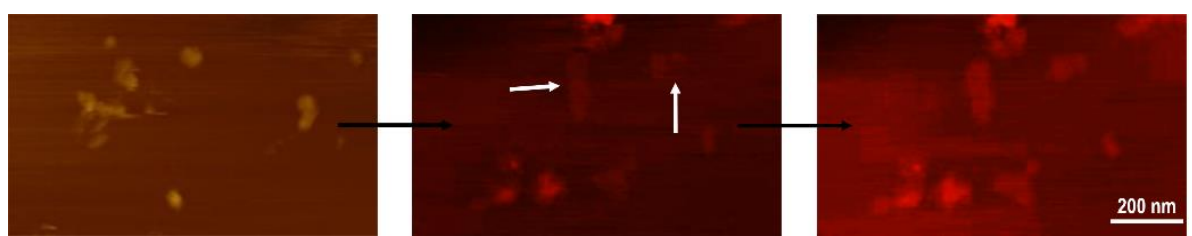

Figure S2. In situ AFM images reveal a dissolution-recrystallization pathway for the formation of HAP. The time step is about 4 min between images. 

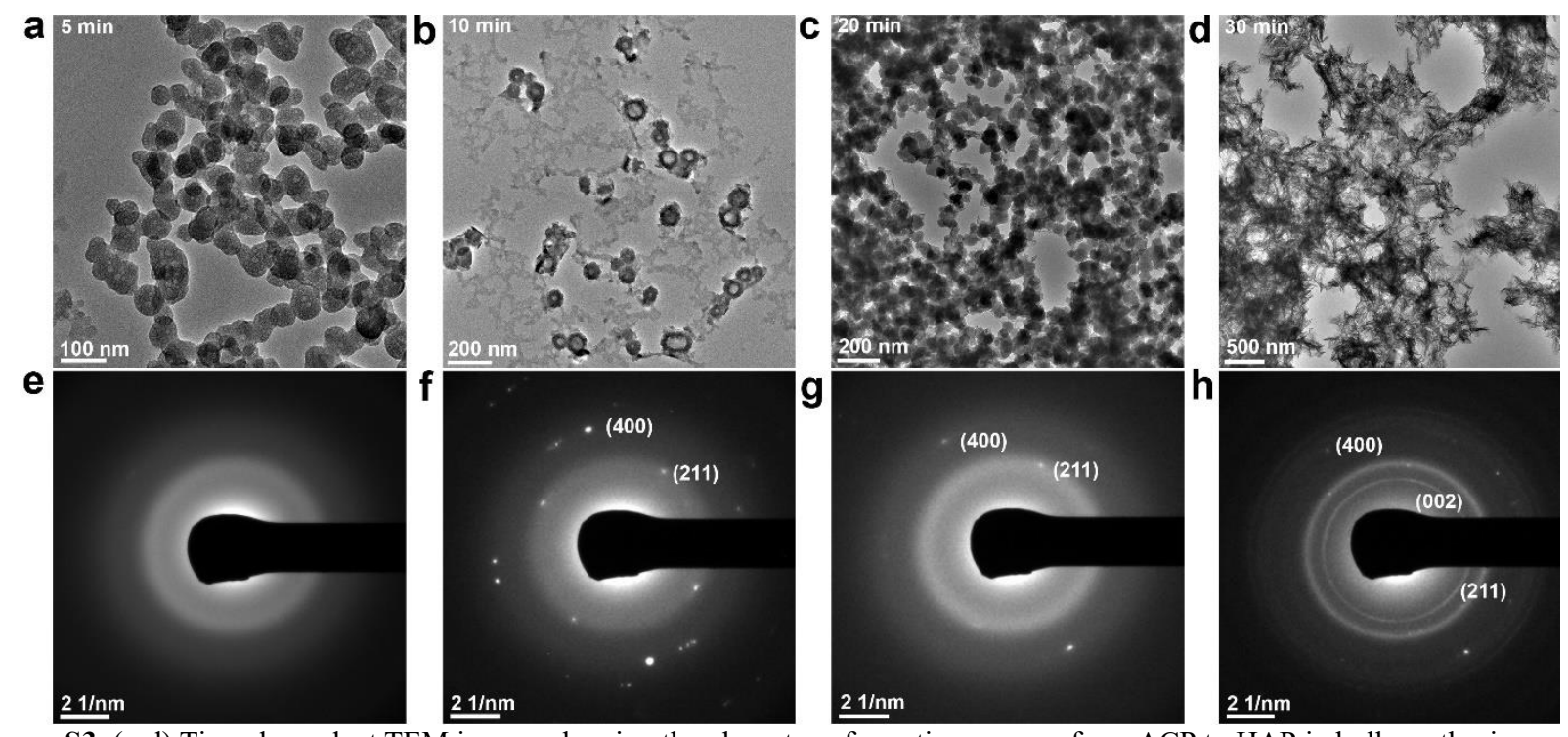

Figure S3. (a-d) Time dependent TEM images showing the phase transformation process from ACP to HAP in bulk synthesis. (e-h) Corresponding SAED data.


Figure S4. TEM image showing ACP and ACP/HAP mixtures in the absence of $\mathrm{Mg}^{2+}$.

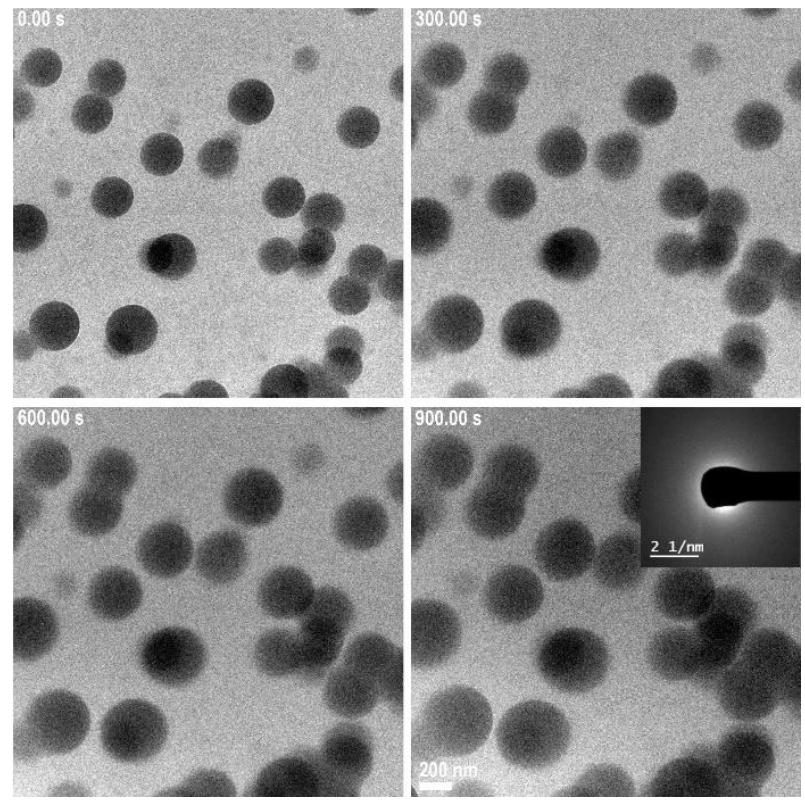


Figure S5. Time series of in situ TEM images showing the growth process of ACP in the liquid-cell.
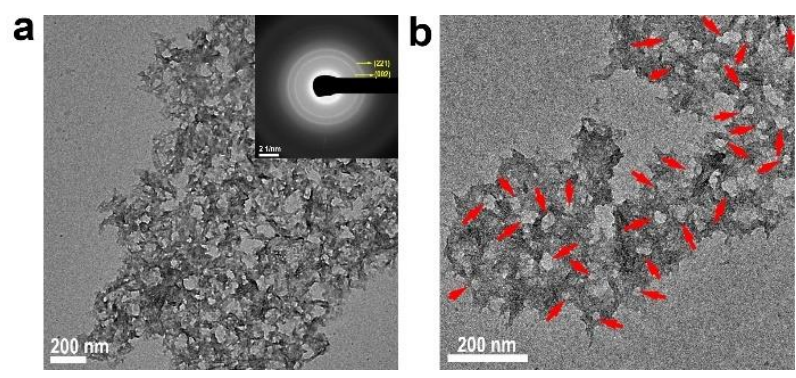

Figure S6. (a, b) TEM images of porous HAP aggregates. Red arrows in (b) mark the locations of pores. Inset to a shows the electron diffraction pattern.
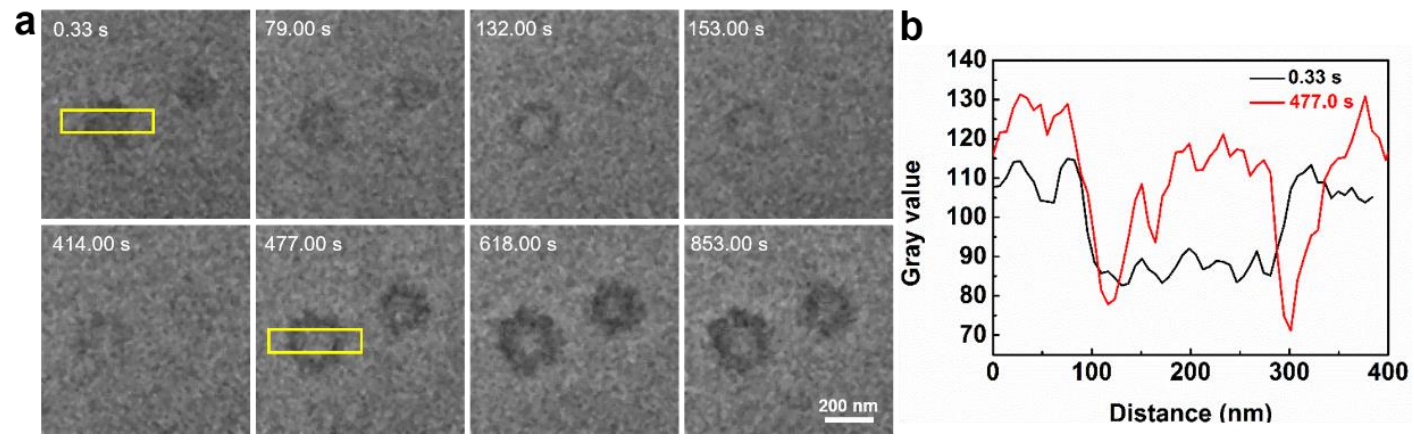

Figure S7. (a) In situ TEM images showing dissolution and recrystallization of ACP particles occurring in two steps. (b) lowercross sections of gray values from the regions in the yellow boxes in (a).




Figure S8. The TEM image of hollow calcium phosphate structures in liquid-cell. Solid ACP nanoparticles with smaller size are highlighted by white arrows.


Figure S9. (a) TEM image of ACP nanoparticles prepared in bulk solution. (b) Size distribution of the particles. 